\title{
Coarse Particle Motion Characteristics in a Double-Stage Slurry Pump Considering Leakage Flow
}

\author{
Hao Jia, Yuqi Wang, Zuchao Zhu, Xianghui Su $\mathbb{D}^{\text {D, }}$, and Zhenji Tang \\ Key Laboratory of Fluid Transmission Technology of Zhejiang Province, Zhejiang Sci-Tech University, Hangzhou 310018, China \\ Correspondence should be addressed to Xianghui Su; suxianghui@zstu.edu.cn
}

Received 8 July 2021; Revised 24 September 2021; Accepted 4 October 2021; Published 28 October 2021

Academic Editor: Antonio Giuffrida

Copyright $\odot 2021$ Hao Jia et al. This is an open access article distributed under the Creative Commons Attribution License, which permits unrestricted use, distribution, and reproduction in any medium, provided the original work is properly cited.

\begin{abstract}
Along with the pressing demand for the long-distance transportation of coarse particles in the deep-sea mining industry, evaluating the slurry pump's passing through and erosive wear by studying the particle motion characteristics and the slurry behavior is becoming increasingly important. Research on the influence of leakage flow through the clearance and balancing devices on the motion characteristic of granular grain flow is of great significance but has been seldom studied. This study coupled the discrete element method with the CFD method to investigate the comprehensive effect of a double-stage slurry pump's main flow and leakage flow on the motion characteristics of particles with a $10 \mathrm{~mm}$ diameter. Results show that the leakage flow occupation in main flow falls from $26 \%-27 \%$ to $8 \%-9 \%$ for the two stages, with the flow rate increasing from $80 \mathrm{~m}^{3} / \mathrm{h}$ to $200 \mathrm{~m}^{3} / \mathrm{h}$. In the first stage with leakage, accumulation of coarse particles was observed at the impeller eye, which should be paid much attention to slurry pumps' operation to eliminate the chance of blockage. In the nonleak situation, although the increment of the average kinetic energy of particles through the impeller is more significant than in the leak case, most of them dissipate primarily by more than $10 \%$ collision in the bowl diffuser. In the leak or nonleak case, the average kinetic energy of particles was more than twice through the first stage but only 1.1 times through the second stage. The selection of stages in the slurry pump design should consider the limitation of particle velocity improvement.
\end{abstract}

\section{Introduction}

The hydraulic transportation of solids by the slurry pump has been widely used in situations where a practical approach is needed to convey granular solids, such as dredging industry, mining industry, and ash transportation in power plants $[1,2]$. The useful life of most slurry transport pumps is limited by the block and erosive wear of the flow passages [3]. Therefore, the conventional design of centrifugal pump has been generally modified to develop a slurry pump, including the enlargement of the wetted passage to eliminate the chance of blockage, use of a robust impeller with a smaller number of vanes, and special sealing arrangements to ensure longer life [4].

The modified design faces a great challenge when obtaining a slurry pump used in extreme environments, such as deep-sea mining (Figure 1). In the deep-ocean mining of polymetallic nodules, which are up to $50 \mathrm{~mm}$ in size and deposited at depths as great as $5000 \mathrm{~m}[6,7]$, the slurry pump is the only power of hydraulic hoisting, and the core parts of flow passage should have an optimized structure with resistance to blockage and wear. Along with the urgent requirement of commercial mining in the deep ocean, improving the reliability and wearability of the slurry pump by studying the particle motion characteristics and the slurry behavior is critical $[2,8]$.

Experimental and numerical studies were performed to determine the effect of particle motion on pump performance and flow fields. Engin and Gur [9] presented empirical correlations to estimate the effect of granular solids on the flow field and pump performance. Liu and Zhu [10] studied the motion of particles with diameters ranging from $1 \mathrm{~mm}$ to $12 \mathrm{~mm}$. They found that the coarse particles are easily concentrated in the back and middle channels, whereas the smaller particles are easy to gather on the suction surface. Tarodiya and Gandhi $[4,11]$ focused on the 


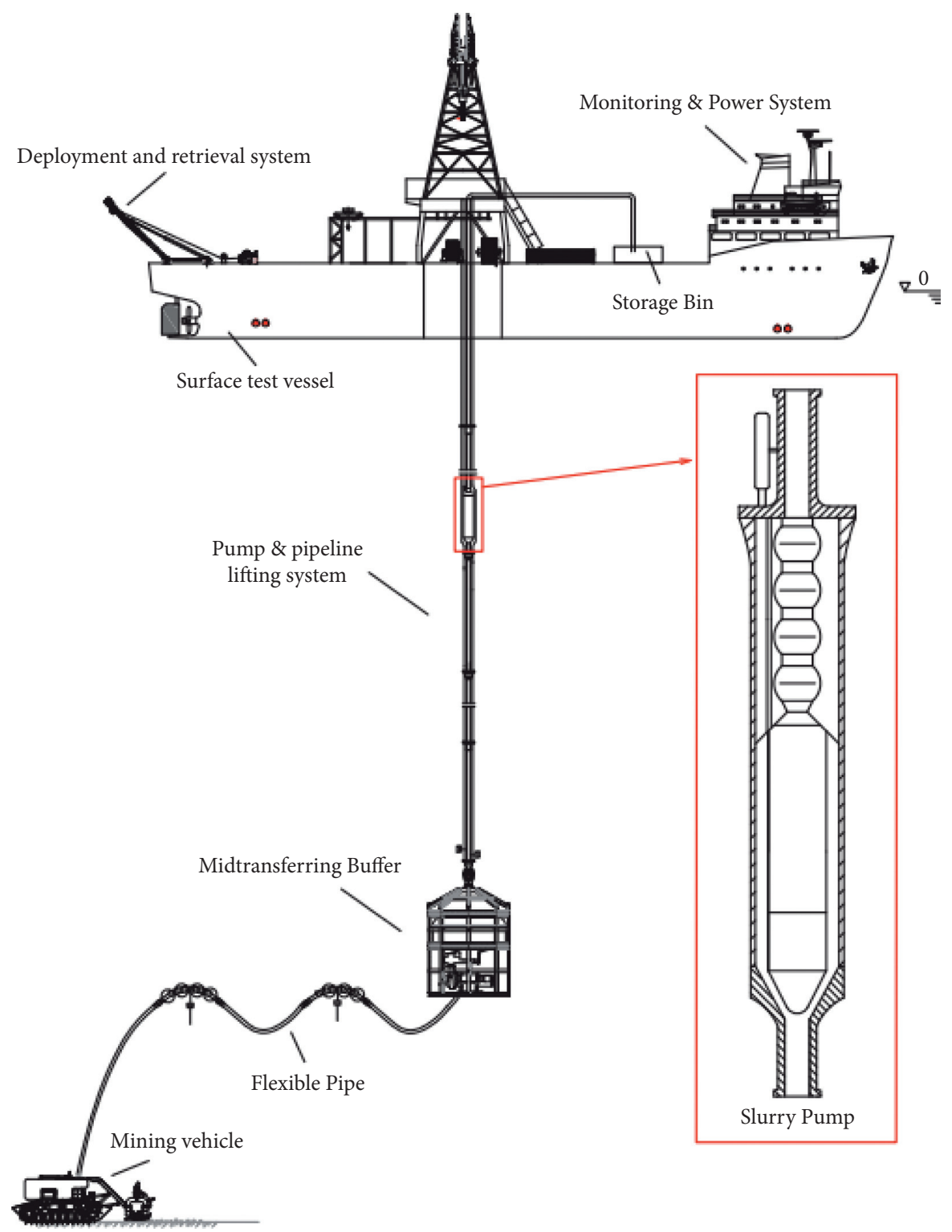

FIgURE 1: Pipeline lifting system of deep-sea mining [5].

erosive wear of the pump casing experimentally and numerically and found the dominant factors affecting the erosion of the pump casing at different operating conditions. Zhang et al. [12] discussed a similar phenomenon by simulating particle trajectory under different particle sizes, volume fractions, and concentrations. Wang et al. [5] studied the movement characteristics and spatial distribution of particles with diameters of 15,10 , and $5 \mathrm{~mm}$ at particle volume concentration of 5\%. They found that the stratification phenomenon gradually disappears with the increase of the intensity and scale of the vortex in the bowl diffuser. Tarodiya and Gandhi [13] also studied the multisized particulate slurry using a granular Eulerian-Eulerian model. They found that the variation in particle size distribution significantly affects the flow of particles inside the impeller and casing flow passages due to particle kinetics.
In the centrifugal pumps, structures with seals and balance holes can effectively balance most of the axial force of the pump by leakage flow. Leakage flow interferes with main flows and causes hydraulic losses. Research on the influence of leakage on the flow characteristics of the pump is of great significance. Until now, the research on clearance flow is mainly concentrated on water pumps. Lei et al. [14] analyzed the performance of a shrouded centrifugal pump for axial clearances with various thicknesses. They found that, as the front clearance increases, the clearance flow more strongly interferes with the main flow, and the backflow at the impeller eye becomes greater. Engin and Gur [15] studied the effect of impeller tip clearance on the performance of a shrouded pump transporting slurry. They found that the volumetric efficiency is most sensitive to the axial clearance variation and has a primary effect on the pump efficiency. Park [16] studied the impact of the back clearance 
size and the balance holes on the backflow of the centrifugal pump with a semiopen impeller and produced empirical equations for the leakage loss through the balance holes using the CFD predictions.

The existing research on slurry pump performance is mainly based on the simplified model without considering the leakage flow, which is quite different from the actual situation. To study the interaction between the tip clearance flow and the main flow, Liu and Tan [17] reported the trajectory of the primary tip leakage vortex (PTLV) and proposed a power function law to describe the spatialtemporal evolution of PTLV. In their following work $[18,19]$, the influences of PTLV, including blade rotation angle, tip clearance size, impeller blade number, and impeller rotational speed, were investigated in detail and the relative vortex stretching item related to velocity gradient was analyzed. Therefore, it is necessary to analyze the transport characteristics and solid behaviors under the comprehensive effect of the main flow and leakage flow. Comparing with the submillimeter particle, which can go through the sealing ring $[4,20]$, coarse particles do not go through the clearance, which leads to wear of the chamber, but the leakage flow influences them. Investigation on motion characteristics of coarse particles in slurry pumps considering leakage flow is vital in providing theoretical and application support for the design and analysis of the slurry pump [21].

In this work, the hydraulic model of a double-stage slurry pump with a balance hole and clearance in front and back chambers was established to investigate the motion characteristics of coarse particles considering leakage flow. The CFD-DEM coupling model was performed to analyze the influence of leakage flow on the main flow and particle motion in different flow rates. The results obtained from the seven special zones clearly show the motion properties of the particle groups. The variation in particle kinetic energy was analyzed through a comprehensive evaluation of the slurry pump's flow characteristics and particle collision.

\section{Materials and Methods}

2.1. Physical Model. The double-stage slurry transport pump with the rotating speed of $1450 \mathrm{r} / \mathrm{min}$ was adopted as the model pump with its designed flow rate and total head of $120 \mathrm{~m}^{3} / \mathrm{h}$ and $40 \mathrm{~m}$, respectively. The hydraulic domain of a single stage includes the inlet duct, front chamber, impeller, back chamber, balance chamber, bowl diffuser, and outlet duct (Figure 2). The front chamber connects the entrance and exit of the impeller with a clearance of $0.5 \mathrm{~mm}$, which was the usual gap in the slurry pump. The same clearance is designed at the back chamber, connecting the balance chamber with the impeller exit. Furthermore, interstage clearance also exists between the two stages (shown in Figure 2). In this model, the geometry of the balance hole is designed to fulfill the request of axial force and volumetric efficiency. The clear schematic of the clearance structure and associated leakage flow are shown in Figure 3 in Results and Discussion (take the second stage as an example). The pump is vertically placed with the inlet at the bottom and the

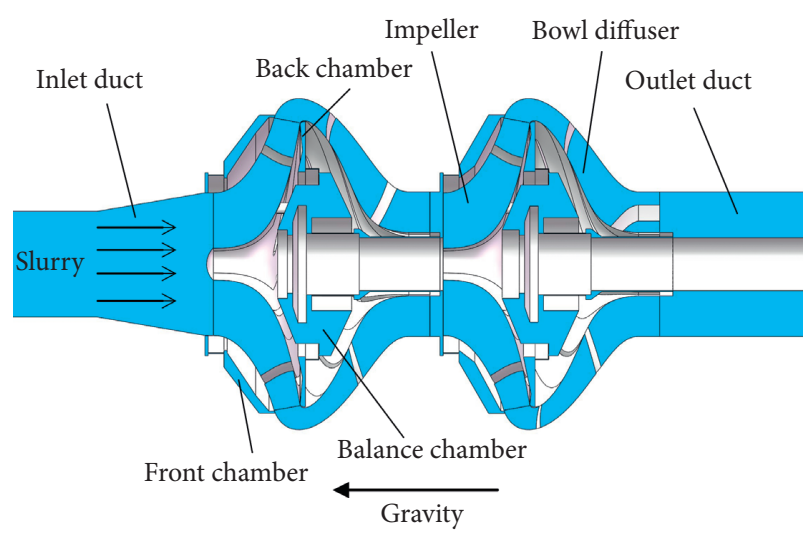

Figure 2: Computational domain of the pump.

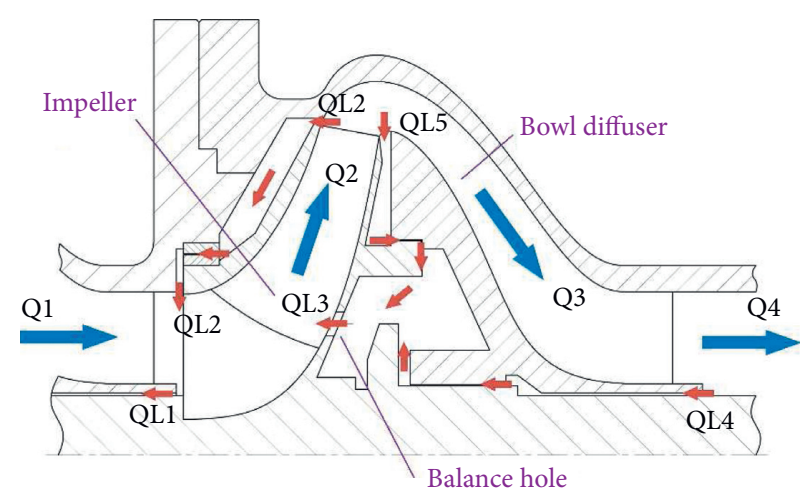

Figure 3: Leakage flow in the second stage of the slurry pump.

gravity was considered in the simulation. Detailed structure parameters of the pump are shown in Table 1.

2.2. Numerical Method. The Euler-Lagrange method was adopted to perform the simulation of the solid-liquid flow in the slurry pump. The computational model employs DEM for simulating the particle dynamics in the two-phase flow in conjunction with the CFD method [23]. The Reynolds-averaged Navier-Stokes approach modeled the liquid (continuous phase):

$$
\begin{aligned}
\frac{\partial \rho}{\partial t}+\frac{\partial}{\partial x_{i}}\left(\rho u_{i}\right) & =0 \\
\frac{\partial}{\partial t}\left(\rho u_{i}\right)+\frac{\partial}{\partial x_{j}}\left(\rho u_{i} u_{j}\right) & =-\frac{\partial P}{\partial x_{i}}+\frac{\partial \tau_{i j}}{\partial x_{j}}+\rho g_{i}+F_{i}
\end{aligned}
$$

where the fluid-particle interaction force $F_{i}$ is calculated by the Morsi-Alexander model [22]:

The translational and rotational motion of the spherical solids (discrete phase) can be described individually as follows:

$$
\begin{aligned}
m_{i} \frac{\mathrm{d} v_{i}}{\mathrm{~d} t} & =\sum\left(F_{n, i j}+F_{\tau, i j}\right)+F_{f p, i}+m_{i} g, \\
\frac{\mathrm{d}}{\mathrm{d} t} I_{i} w_{i} & =\sum\left(r_{i} \times F_{\tau, i j}+M_{i}\right) .
\end{aligned}
$$


TABLE 1: Geometric parameters for the model slurry transport pump [22].

\begin{tabular}{lccc}
\hline Impeller parameters & Value & Bowl diffuser parameters & Value \\
\hline Inlet diameter $(\mathrm{mm})$ & 135 & Inlet diameter $(\mathrm{mm})$ & 276 \\
Outlet diameter $(\mathrm{mm})$ & 272 & Inlet width $(\mathrm{mm})$ & 26 \\
Outlet width $(\mathrm{mm})$ & 25 & Axial length $(\mathrm{mm})$ & 127.5 \\
Blade number & 4 & Blade number & 5 \\
Wrap angle $\left({ }^{\circ}\right)$ & $111-115$ & Wrap angle $\left({ }^{\circ}\right)$ & 118 \\
Outlet angle $\left({ }^{\circ}\right)$ & 24 & Outlet angle $\left({ }^{\circ}\right)$ & 25 \\
Inlet angle $\left({ }^{\circ}\right)$ & 26 & Inlet angle $\left(^{\circ}\right)$ & 90 \\
\hline
\end{tabular}

The spherical particles with $5 \%$ were set as the inlet boundary condition in the simulation. The contact forces $F_{\tau, i j}$ were solved by the Hertz-Mindlin model [24], and the related coefficients of friction and restitution used in the model can be referenced to [23]. The material of pump body and particle are alloy and polymetallic nodules, respectively [22], whose properties are shown in Table 2 [24].

This study coupled Fluent 18.0 and EDEM 2018 to perform the simulation. The shear stress transport $k-\varepsilon$ model with standard wall function was used to simulate the slurry flow in the pump because of its good accuracy and better prediction for flow separation [25]. The mass flow inlet and free outflow conditions were adopted at the inlet and outlet boundaries, respectively. The influence of external excitation from the ocean wave is ignored in our simulation. Therefore, a nonslip boundary condition was set for all the walls. Time step of the fluid and particle function is set as $10^{-4} \mathrm{~s}$ and $5 \times 10^{-5} \mathrm{~s}$.

The convergence residual was set as $10^{-5}$. Combined with the monitor of the residual error feature of pump outlet pressure, we also monitor the instantaneous particle count (volume fraction of particles) in the domain. When the count stays nearly as a constant, the simulation is considered to be stable.

2.3. Grid and Computing Settings. In this paper, the particle diameter was chosen as $10 \mathrm{~mm}$, which was closely related to the request in deep-ocean mining. The cell size of the particle-passing components was adopted no more than the particle diameter $(10 \mathrm{~mm})$ to avoid nonphysical and nonsmooth results in the CFD-DEM simulation [5]. Figure 4 shows the computational grids of the slurry transport pump. In the nonleak case, four sets of meshes from elements of 130,354 to 219,408 are adopted to conduct the calculation. As shown in Table 3, the difference in the head, efficiency, and pressure (located on the blade suction side) falls below $4 \%$ when the grid number exceeds 180,000 . Therefore, grid number 191,111 was adopted in the nonleak case (Figure 4(a)). In the leak case, fine mesh is adopted at the clearances and cavities to generate four sets of meshes while meshes in other parts remain unchanged (Figure 4(b)). The variation of pressure (located in the back chamber) falls below $3 \%$ when the grid number exceeds 720,000 and the grid number 722,614 was finally adopted.

2.4. Experimental Validation. The hydraulic performance of the model pump was tested in the slurry transport system
(Figure 5) to validate the model adopted in this study. The system consists of two parts: the circulating ring and the pressure ring (marked in red and blue arrows, respectively). The pressure ring was designed to adjust the pump's outlet pressure by adjusting the valve before the water tank (blue fort). The pump inlet and outlet pressures were measured by pressure gauges. The flow rates were measured by an electromagnetic flowmeter. The measurement uncertainty is less than $\pm 0.5 \%$. The slurry pump in the experiment is in leak.

Figure 6 compares the leak situation between numerical simulation and experiments in the water and slurry condition. Overall, the predicted efficiency, head, and shaft power errors in water and slurry conditions were all within $3.7 \%$ of the experimental data. Thus, the results can reasonably predict the pump performance. Figure 3 also shows the hydraulic performance of the pump without leakage. Given volumetric loss, the head and efficiency of the leak situation are less than those of a nonleak situation.

\section{Results and Discussion}

3.1. Flow Features Influenced by Leakage Flow. The leakage flow in the two-stage slurry pump can be illustrated by the diagram of the second stage in Figure 3. Q1, Q2, Q3, and Q4 are the main flow at the exit of the first stage, in the impeller, in the bowl diffuser, and at the pump outlet, respectively. QL1, QL2, QL3, QL4, and QL5 are flows of the interstage leakage from the first stage, front chamber leakage, back chamber leakage, balance hole leakage, and interstage leakage from the second stage, respectively. All the leakage flow comes back to the impeller passage through the clearance or the balance, leading to complex flow in the impeller. The leakage flow in the first stage is similar to the second stage, except for the interstage leakage at the impeller eye. Q2 and Q3 can be calculated by

$$
\begin{aligned}
& \mathrm{Q} 2=\mathrm{Q} 1+\mathrm{Q} L 2+\mathrm{Q} 23-\mathrm{Q} 1, \\
& \mathrm{Q} 3=\mathrm{Q} 2-\mathrm{Q} 2-\mathrm{QL5}, \\
& \mathrm{QL}=\mathrm{QL2}+\mathrm{QL3}+\mathrm{QL4}+\mathrm{QL} 5 \sqrt{b^{2}-4 \mathrm{ac} .}
\end{aligned}
$$

Figure 7 shows the velocity distribution in the meridional plane of the pump. The maximum velocity appears at the impeller's exit, near the leakage region to the front and back chamber. Without volumetric loss, the flow progresses more smoothly in the impeller, with a higher velocity at the 
TABLe 2: Properties of particle and pump body.

\begin{tabular}{lcccc}
\hline & Poisson's ratio & Shear modulus (MPa) & Density & Diameter \\
\hline Particle & 0.4 & 21.4 & $2000 \mathrm{~kg} / \mathrm{m}^{3}$ & $10 \mathrm{~mm}$ \\
Pump body & 0.3 & 70 & $7800 \mathrm{~kg} / \mathrm{m}^{3}$ & - \\
\hline
\end{tabular}

TABLE 3: Grid independence test.

\begin{tabular}{|c|c|c|c|c|c|}
\hline & No. & Cells & $H / H_{1}$ & $\eta / \eta_{1}$ & $p / p_{1}$ \\
\hline \multirow{4}{*}{ Nonleak case } & 1 & 130,354 & 1.000 & 1.000 & 1.000 \\
\hline & 2 & 164,687 & 0.976 & 0.983 & 0.985 \\
\hline & 3 & 191,111 & 0.969 & 0.975 & 0.976 \\
\hline & 4 & 229,408 & 0.965 & 0.971 & 0.972 \\
\hline \multirow{4}{*}{ Leak case } & 1 & 484,378 & 1.000 & 1.000 & 1.000 \\
\hline & 2 & 621,314 & 0.965 & 0.971 & 0.983 \\
\hline & 3 & 722,614 & 0.966 & 0.971 & 0.977 \\
\hline & 4 & 819,244 & 0.965 & 0.971 & 0.974 \\
\hline
\end{tabular}

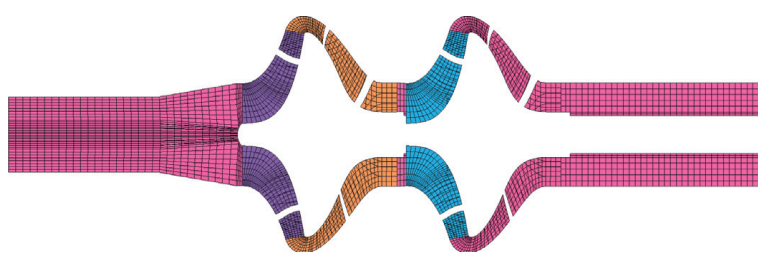

(a)

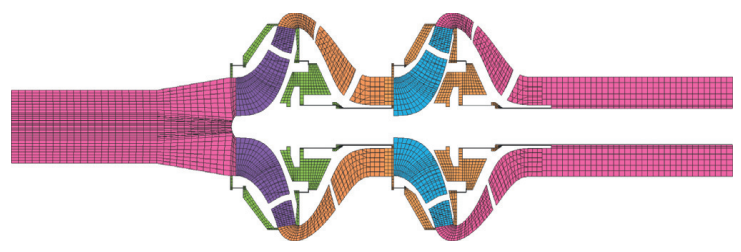

(b)

Figure 4: Computational grids of slurry transport pump: (a) non-leak case and (b) leak case.

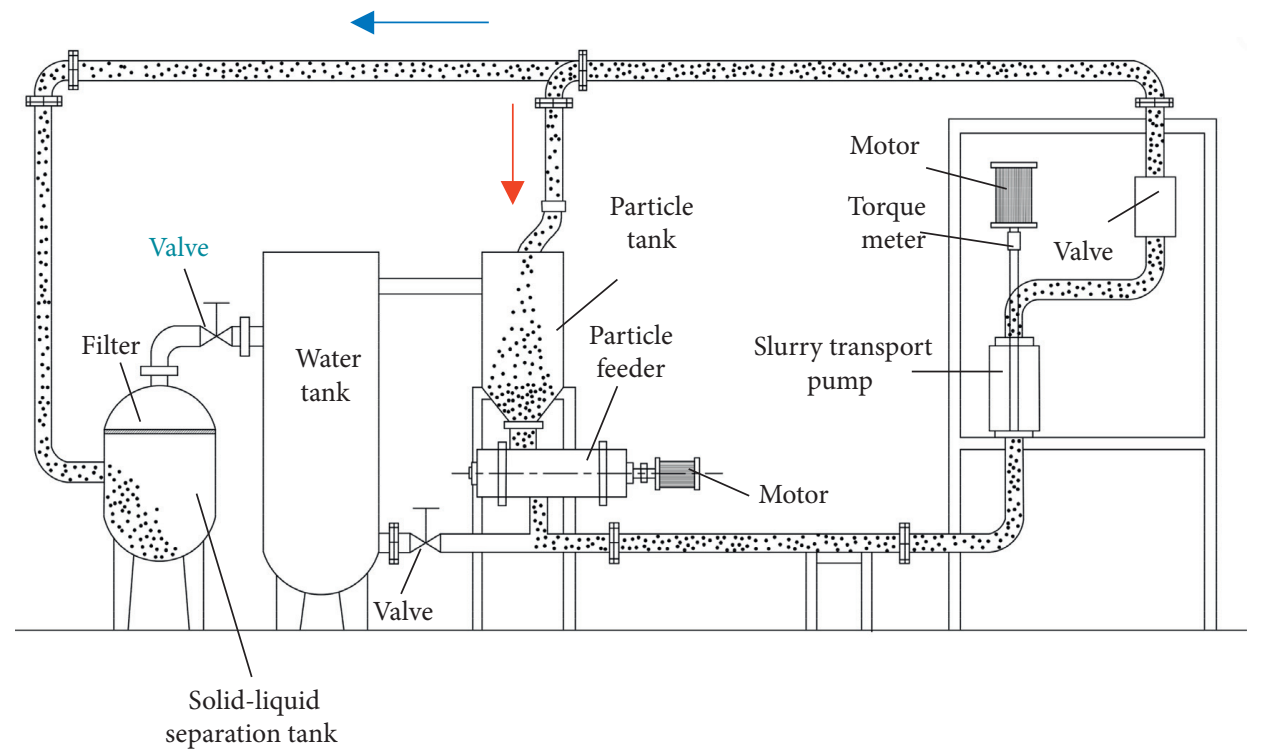

Figure 5: Schematic diagram of experimental apparatus of the slurry transport system.

entrance of the bowl diffuser. In the first stage's impeller, given the squeeze of the leakage from the balance hole, the streamline deviates to the shroud, and a vortex is at the hub side of the impeller exit. The effect of the balance chamber leakage to the main flow at the second stage is less than that in the first stage, and the vortex is not observed at the impeller's exit. Therefore, the axial clearance has an important effect on the internal flow structures in the slurry pump.
The leakage flow was counted under different flow rates based on equations (1)-(2) (Figure 8) to reveal the relative sizes of leakage flows in a different position. Given low flow resistance, $Q L 2$ and $Q L 3$ are larger than $Q L 4$ and $Q L 5$. The interstage $Q L 4$ is the minimum leakage flow because the pressure difference between the bowl diffuser exit and the balance chamber is the smallest. Figure 8 shows that QL2, $Q L 3$, and $Q L 5$ decrease with the increase of flow rates. The 

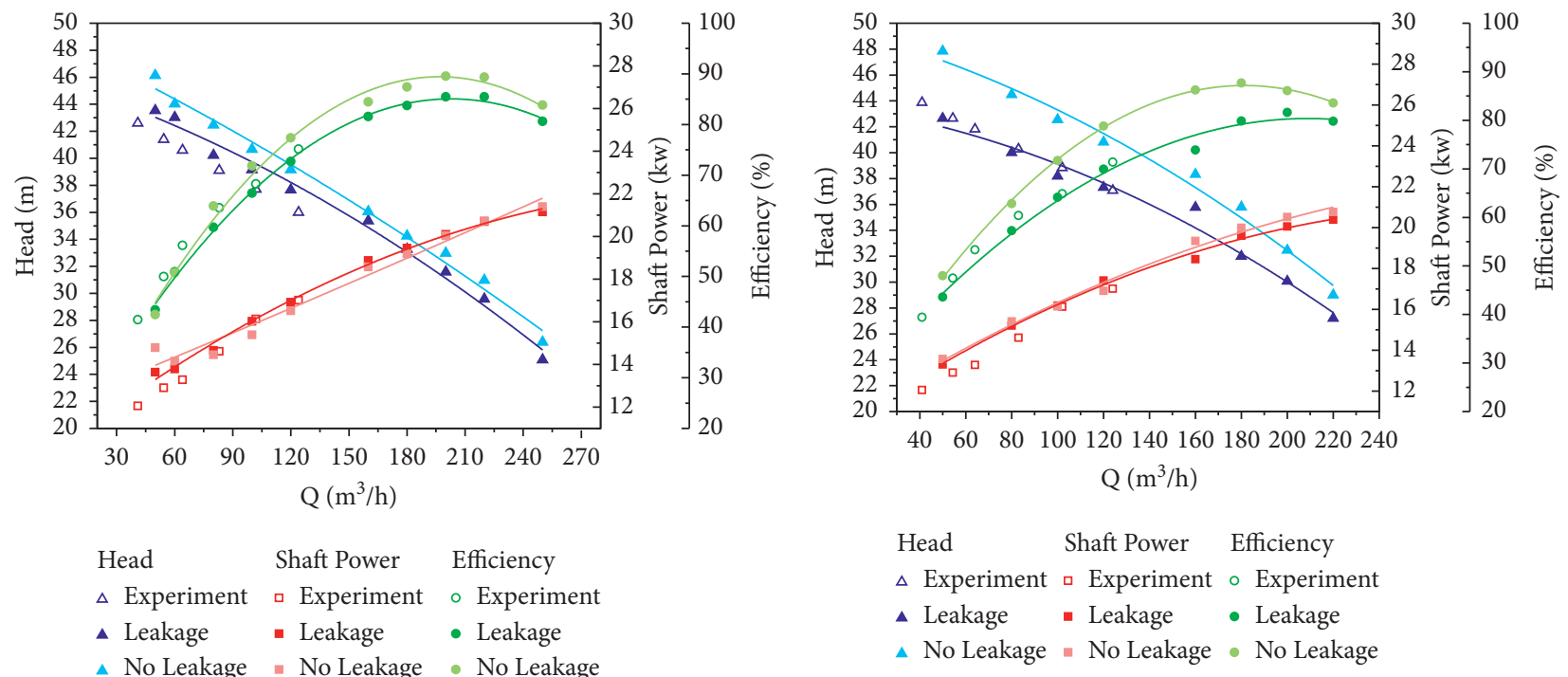

(b)

FiguRE 6: Comparison of the hydraulic performance between experiment and simulation: (a) water condition and (b) slurry condition.
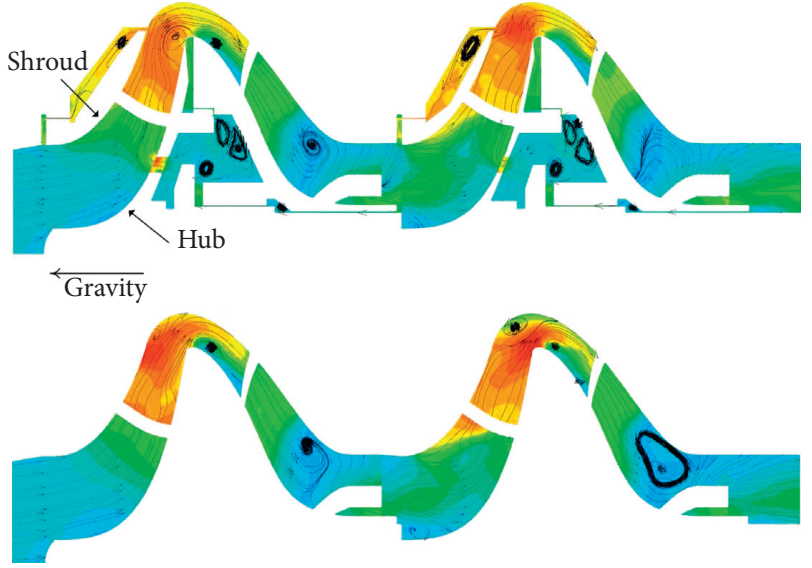

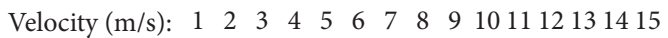

Figure 7: Velocity of the fluid in the meridional plane at $1.2 \mathrm{~s}$ of pumps with or without leakage: (a) leak situation and (b) nonleak situation.

greater the flow rate, the more pressure difference was balanced by the centrifugal force of liquid rotation. The interstage leakage is the flow in the axial direction, which was less influenced by the centrifugal force. Therefore, $Q L 4$ is less affected by the flow rates.

The ratio of leakage flow to the total flow at different flow rates is shown in Table 4. In the first stage, QL2 and QL3 are almost the same at different flow rates. In the second stage, a $1.2 \%$ difference is observed between $Q L 3$ and $Q L 2$ at $80 \mathrm{~m}^{3} / \mathrm{h}$. The $Q L / Q$ falls from $26 \%-27 \%$ to $8 \%-9 \%$ for the two stages, with the flow rate increasing from $80 \mathrm{~m}^{3} / \mathrm{h}$ to $200 \mathrm{~m}^{3} / \mathrm{h}$. The difference reduces gradually with the flow rate.

Figure 8 and Table 4 show that $Q L$ or $Q L / Q$ decreases with $Q$. The reason is that the more significant the flow rate in the main flow, the less pressure difference that causes

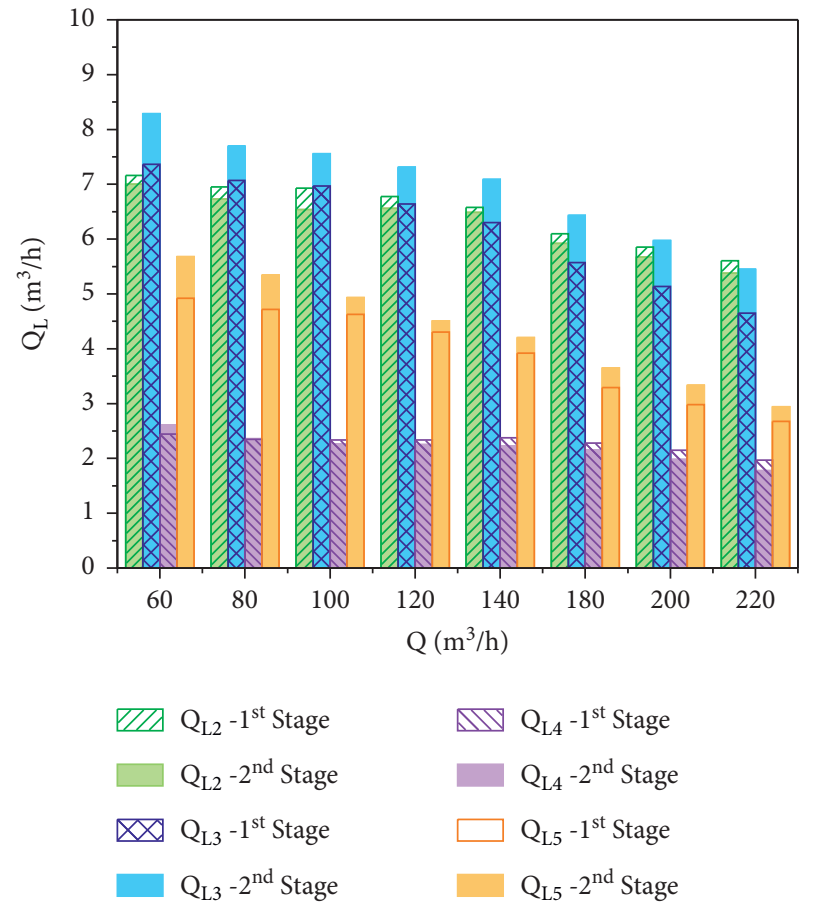

FIGURE 8: Leakage flow of the two stages under different flow rates.

leakage. The effect of the flow on the kinetics of particles is discussed below.

3.2. Distribution and Motion of Particles. The energy transported from the pump provides the kinetics of the slurry (the particles and the water). Generally, the better the particles follow the fluid, the more kinetics transported to the particles. Leakage flow influences the spatial distribution and the motion of the particles. 
TABLe 4: Ratio of leakage flow to the total flow at different flow rates.

\begin{tabular}{|c|c|c|c|c|c|c|c|c|c|c|}
\hline \multirow{2}{*}{$\mathrm{Q} /\left(\mathrm{m}^{3} / \mathrm{h}\right)$} & \multicolumn{5}{|c|}{ First stage } & \multicolumn{5}{|c|}{ Second stage } \\
\hline & QL2/Q & QL3/Q & QL4/Q & QL5/Q & $\mathrm{QL} / \mathrm{Q}$ & QL2/Q & QL3/Q & QL4/Q & QL5/Q & $\mathrm{QL} / \mathrm{Q}$ \\
\hline 80 & $8.7 \%$ & $8.8 \%$ & $3.0 \%$ & $5.9 \%$ & $26.4 \%$ & $8.4 \%$ & $9.6 \%$ & $3.0 \%$ & $6.7 \%$ & $27.7 \%$ \\
\hline 120 & $5.6 \%$ & $5.5 \%$ & $1.9 \%$ & $3.6 \%$ & $16.6 \%$ & $5.5 \%$ & $6.1 \%$ & $1.9 \%$ & $3.8 \%$ & $17.3 \%$ \\
\hline 160 & $3.9 \%$ & $3.9 \%$ & $1.5 \%$ & $2.5 \%$ & $11.8 \%$ & $3.9 \%$ & $4.1 \%$ & $1.4 \%$ & $2.7 \%$ & $12.1 \%$ \\
\hline 200 & $2.9 \%$ & $2.6 \%$ & $1.1 \%$ & $1.5 \%$ & $8.1 \%$ & $2.8 \%$ & $3.0 \%$ & $1.0 \%$ & $1.7 \%$ & $8.5 \%$ \\
\hline
\end{tabular}

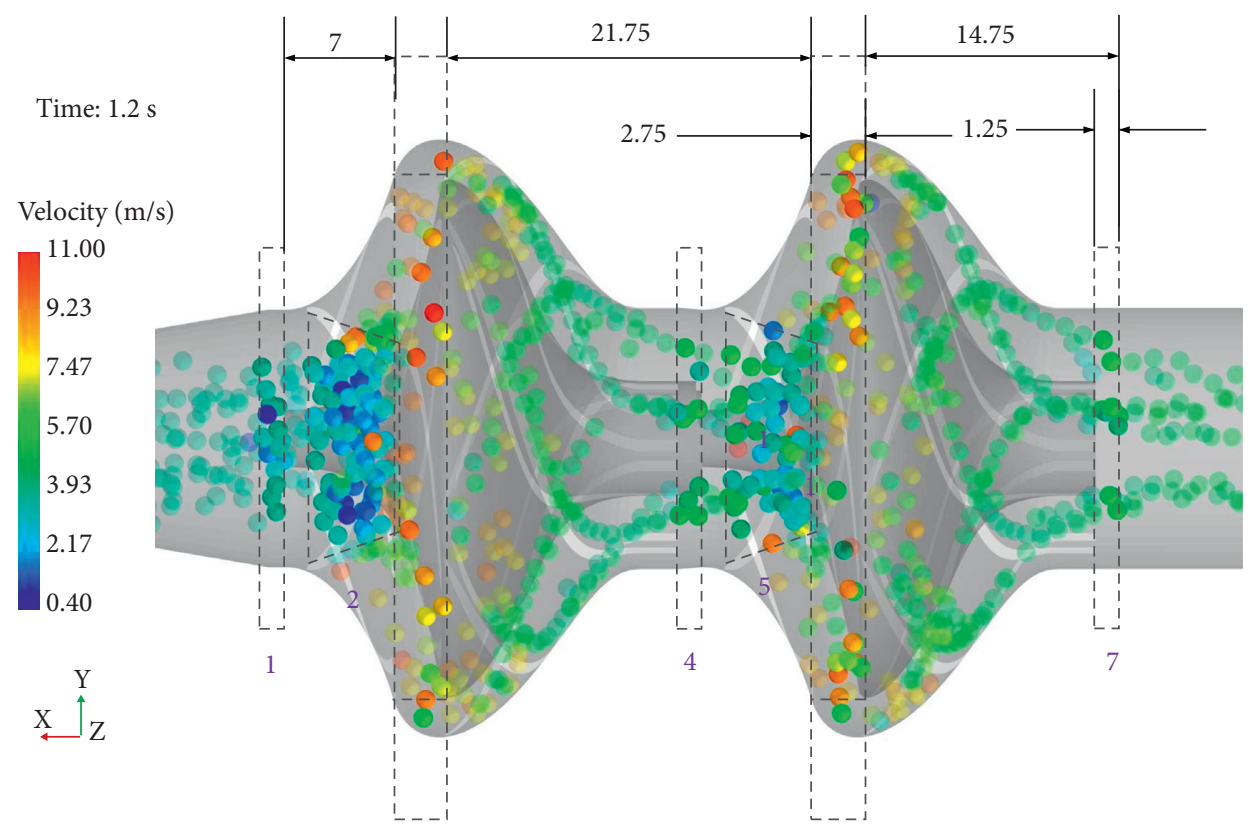

(a)

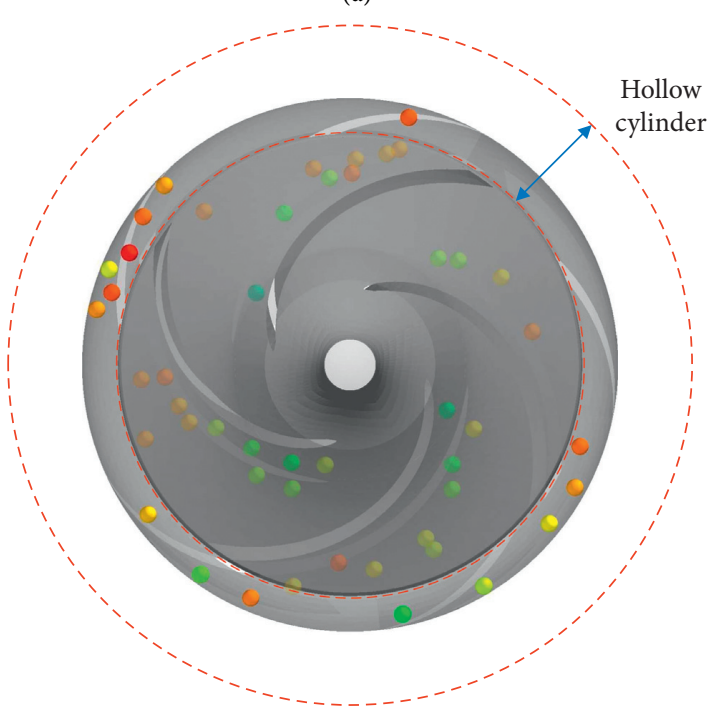

(b)

FIGURE 9: Distribution and velocity of particle groups at $1.2 \mathrm{~s}$ with leakage. The velocity magnitude of the particles is marked as semitransparent color spheres, among which seven important regions are emphasized as nontransparent spheres: (a) schematic diagram of partition of the pump and (b) front view of Region 3 or 6 shaped as a hollow cylinder. 
Figure 9 shows the velocity distribution of the particles in the pump with leakage. Particles accumulate in the impeller eye. The particle groups in the inlet plane are randomly distributed because of their stable state after long rising in the deep-ocean piping system before flowing into the pump. On the whole, the velocity of particles decreases in the bowl diffuser and at the impeller eye and increases in the impeller's passage.

Seven zones are divided at the inlet (nos. 1 and 4), the deceleration region (nos. 2 and 5), the interface of impeller and bowl diffuser (nos. 3 and 6), and the outlet (nos. 4 and 7) of each stage (Figure 9(a)) to show the motion properties of the particle groups in the important region. Among them, zones 1, 4, and 7 are the cylinder region with a thickness of $1.25 \mathrm{~cm}$. The deceleration region was formed by rotating the leading edge of the impeller around the central axis with a thickness of $7 \mathrm{~cm}$. Zones 3 and 6 are shaped as a hollow cylinder with a thickness of $2.75 \mathrm{~cm}$ to capture the particles at the interface of the impeller and bowl diffuser precisely (Figures 9(a)-9(b)). Figure 10 shows the statistics of the average kinetic energy $K_{j}$ in each area within 1.0-1.2 s, which is calculated as follows:

$$
K_{j}=\frac{\sum_{i=1}^{N} K_{i j}}{N_{j}}(j=1-7),
$$

where $i$ is the particle index and $N_{j}$ is the total number of particles passing through the $j$ area within 1.0-1.2 s.

Given the low inlet speed of the slurry, the average kinetic energy shows a slight decrease at the deceleration region. The velocity of particles increases drastically after rotating with the impeller. In the bowl diffuser, particles change motion direction by colliding with the stationary wall, in the price of dissipation of kinetic energy. In the nonleak situation, the average kinetic energy increases to $39 \mathrm{~mJ}$ and $30 \mathrm{~mJ}$ of the first and second stage, $130 \%$ and $104 \%$ of that in the leak situation. In the slurry pump, fluid suspension and granular contact are two mechanisms of particle support [2]. The fluid suspension mainly characterized by hydraulic lift forces is significant to the motion of larger particles. Granular contact includes flows of coarse particles which travel entirely as contact load and also the contact load component of heterogeneous flows. The forcebalance analysis is difficult to perform to determine the effect of the two mechanisms. This work focuses on the statistical properties of particles when advected by the turbulence in the slurry pump. The number of granular contact in the impeller is shown in Table 5. From Table 5, we can infer that although the collision count in the first impeller is slightly smaller than that in the second stage, the fluid suspension is stronger in the first stage to improve particles' kinetic energy. The law of transformation of velocity magnitude and direction in the impeller is discussed in Section 3.3.

Particles acquire more kinetic energy in the nonleak situation. However, the energy dissipation in the bowl diffuser is also more. It causes lower kinetic energy at the exit of the bowl diffuser than that in the leak situation because of the more frequent particle-particle and particle-wall collision in the bowl diffuser. As shown in Table 5, the collision count in

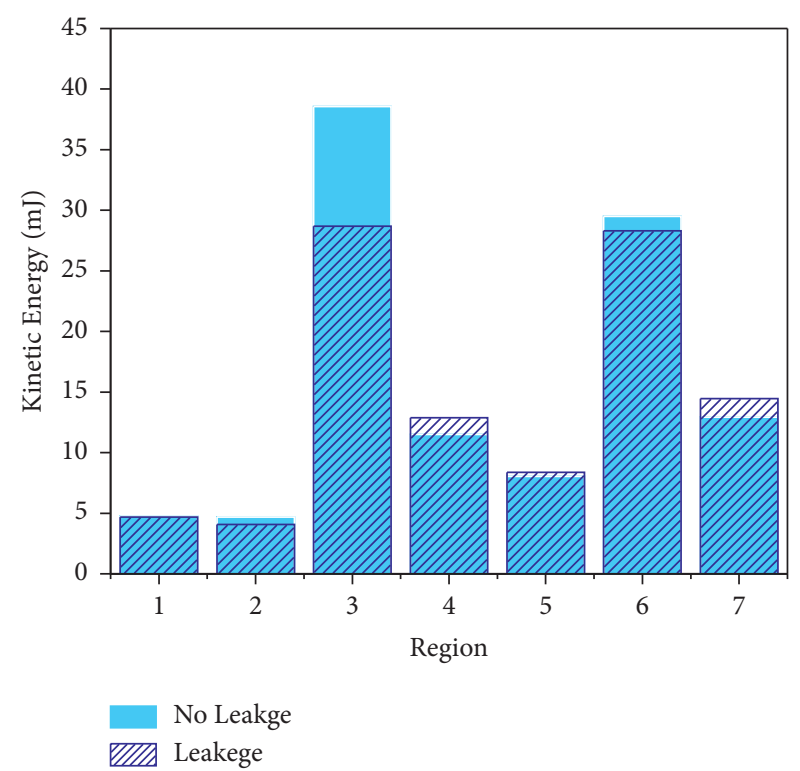

FIGURE 10: Average kinetic energy of particles in each region in a leak and nonleak situation.

TABLE 5: Total collision count within 1.0-1.2 s of the simulation.

\begin{tabular}{lccc}
\hline \multicolumn{1}{c}{ Without leakage } & \multicolumn{2}{c}{ With leakage } \\
\hline First impeller & 951 & First impeller & 1115 \\
First bowl diffuser & 5594 & First bowl diffuser & 5084 \\
Second stage & 1026 & Second stage & 1095 \\
Second bowl diffuser & 5707 & Second bowl diffuser & 4896 \\
\hline
\end{tabular}

the first bowl diffuser without leakage is 5594, which is $10 \%$ more than that with leakage (5084). On the contrary, the collision number is 5707 and 4896 of the leakage and no leakage case, with the relative difference of $16 \%$.

Frequent and intense collisions reduce most of the kinetic energy acquired in the impeller. The average kinetic energy of particles increased by 2.7 times and 2.4 times through the first stage (from Region 1 to Region 4) in leak and nonleak situations, respectively. The difference is much less significant when particles go through the second stage (both are 1.1 times).

Therefore, for the vertical lifting of the slurry, the average kinetic energy of particles slightly increases through the second or following stage in the multistage pump. The choice of stages in the slurry pump design should consider the limitation of particle velocity improvement.

\subsection{Effect of Leakage on Particle Migration in the Impeller.} The distribution of particles in the maximum cross section of the impeller at $1.2 \mathrm{~s}$ at $Q=Q_{d}$ is shown in Figure 11 to show clearly the flow characteristics of particles in the impeller. According to the distribution and velocity of particles, the impeller in the first stage can be divided into two parts, the accumulation region and the accelerating region, which are consistent with that illustrated in Figure 9. Carried by the fluid, particles enter into the impeller and are confronted with the velocity direction 
Time: $1.2 \mathrm{~s}$
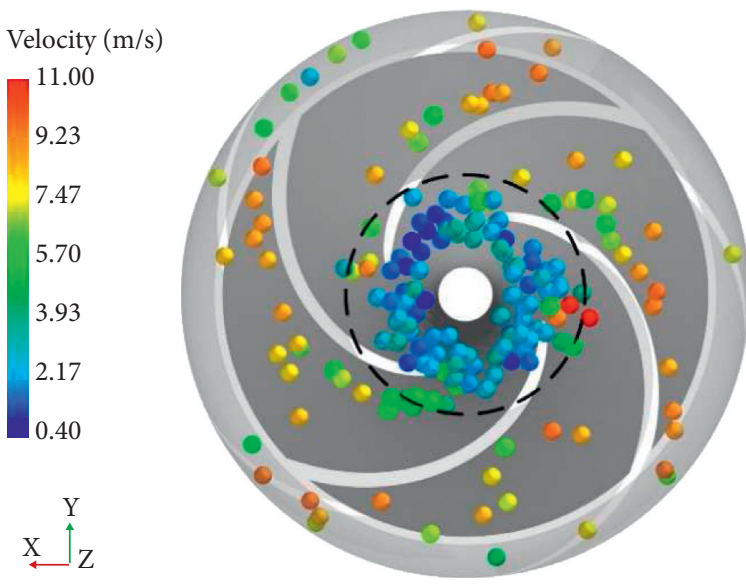

(a)

Time: $1.2 \mathrm{~s}$

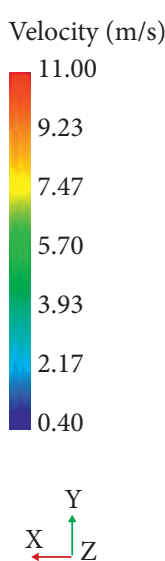

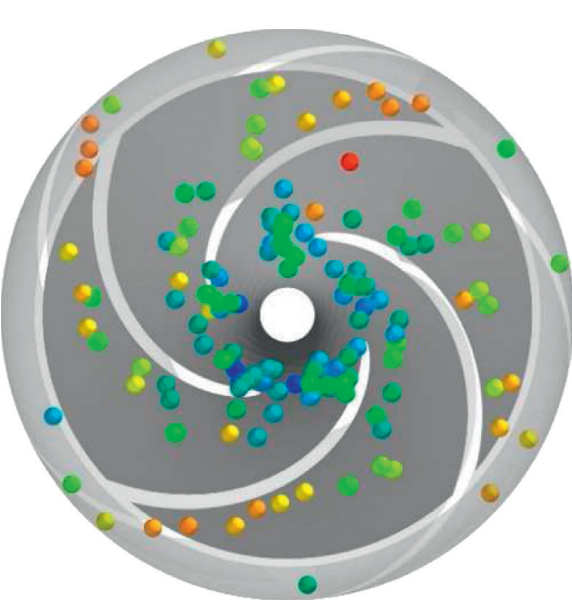

(c)
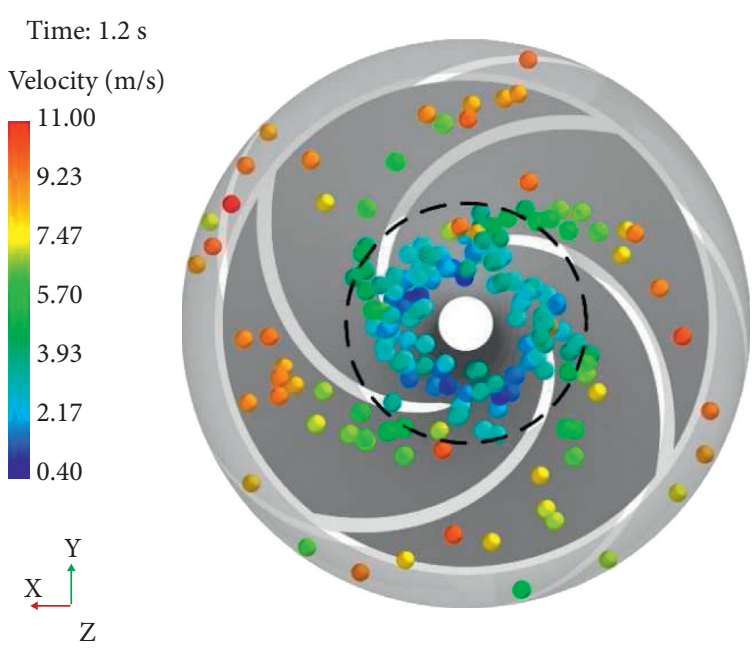

(b)

\section{Time: $1.2 \mathrm{~s}$}

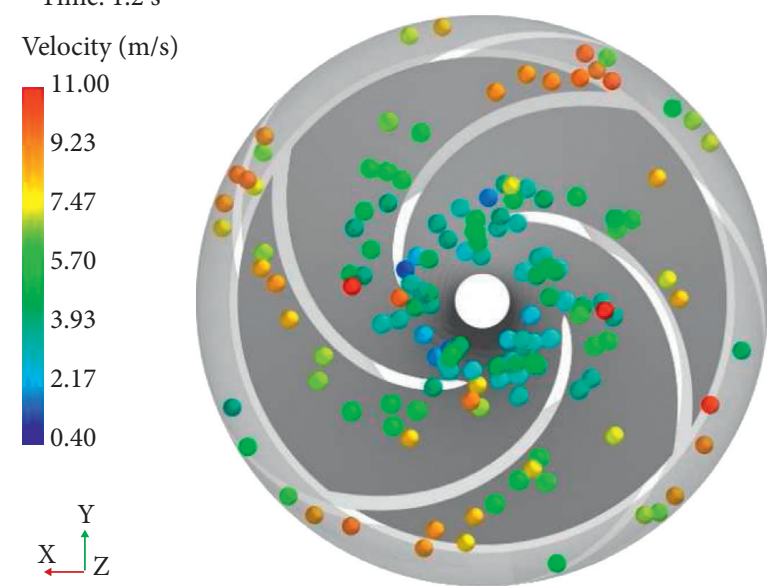

(d)

Figure 11: Distribution of particles in the cross section of the impeller at $1.2 \mathrm{~s}$ at $Q=Q_{d}$ with or without leakage (the black circle marks the accumulation region in the first stage): (a) leakage, first stage; (b) no leakage, first stage; (c) leakage, second stage; (d) no leakage, second stage.

transformation from axial to radial. However, the coarse particles with high inertia spend more time on the variation of motion direction. The leading particles collide with the impeller hub and slow down. They may rebound to collide with the following particles, forming the accumulation region. The radial velocity of particles reduces and accelerates in the radial direction. Comparing with the nonleak situation, the impeller with leakage losses part of the fluid kinetic energy (Figure 7). Therefore, the particles tend to accumulate in the impeller eye.

In the accelerating region, particles speed up and become sparse in distribution. Huang et al. found that [23] small particles with a diameter of $1 \mathrm{~mm}$ to $3 \mathrm{~mm}$ have good following performance with the fluid, dominantly move adjacent to the pressure sides of the impeller vanes, and tend to deviate from the pressure sides near about onethird of the length of the vanes while moving downstream. Particles, with a diameter of $10 \mathrm{~mm}$ in this study, collide with the blade of the impeller, which has a larger linear speed, and then move close to but not adjacent to the pressure side of the impeller. The large inertia of coarse particles lowers its following behavior to the continuous phase. Thus, moving close to the pressure surface of the blades is hard.

In the second stage, higher axial velocity of the particles at the inlet of the impeller leads to a faster transformation of velocity from axial to radial. Therefore, the interface between the accumulation region and the accelerating region is not apparent (Figures 11(c) and 11(d)).

In Figure 12, most of the velocity of particles in the accumulation region is less than $4 \mathrm{~m} / \mathrm{s}$. The particle count is much larger in nonleak cases than in leak cases in the $0-2 \mathrm{~m} / \mathrm{s}$ region, reflecting the effect of leakage on particle aggregation.

To illustrate the characteristics of velocity in the impeller quantitively, Figure 13 shows the velocity distribution versus particle radial position $R_{p}$. In the accumulation region $\left(R_{p}<6 \mathrm{~cm}\right)$, particles accumulate with $V_{p}$ less than $4 \mathrm{~m} / \mathrm{s}$. In the accelerating region $\left(R_{p}>6 \mathrm{~cm}\right)$ of the nonleak case, $V_{p}$ increases almost 


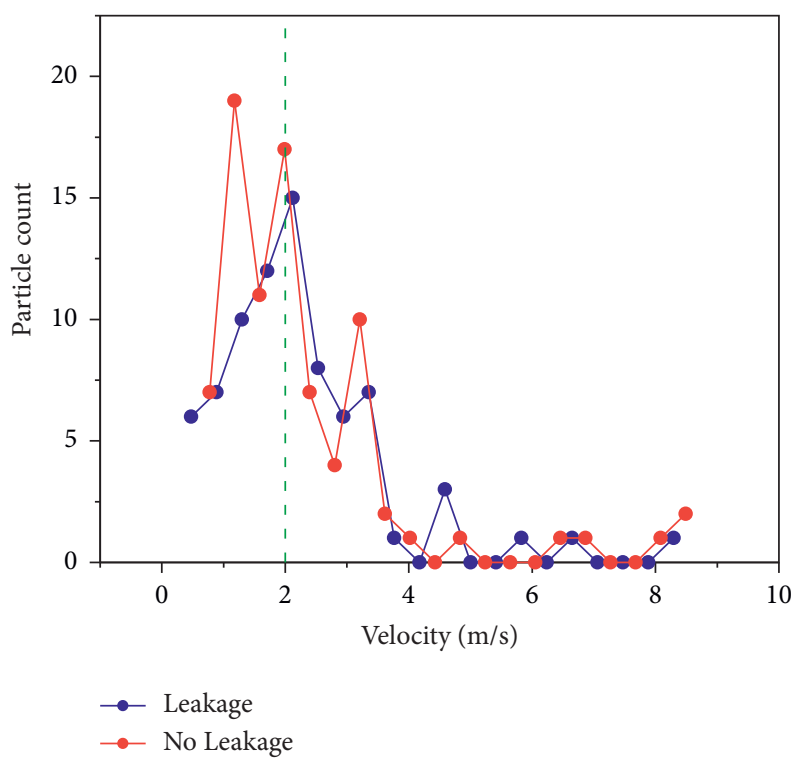

FIGURE 12: Distribution of particle velocity in the accumulation region of the first-stage impeller at $1.2 \mathrm{~s}$ at $Q=Q_{d}$ with or without leakage.

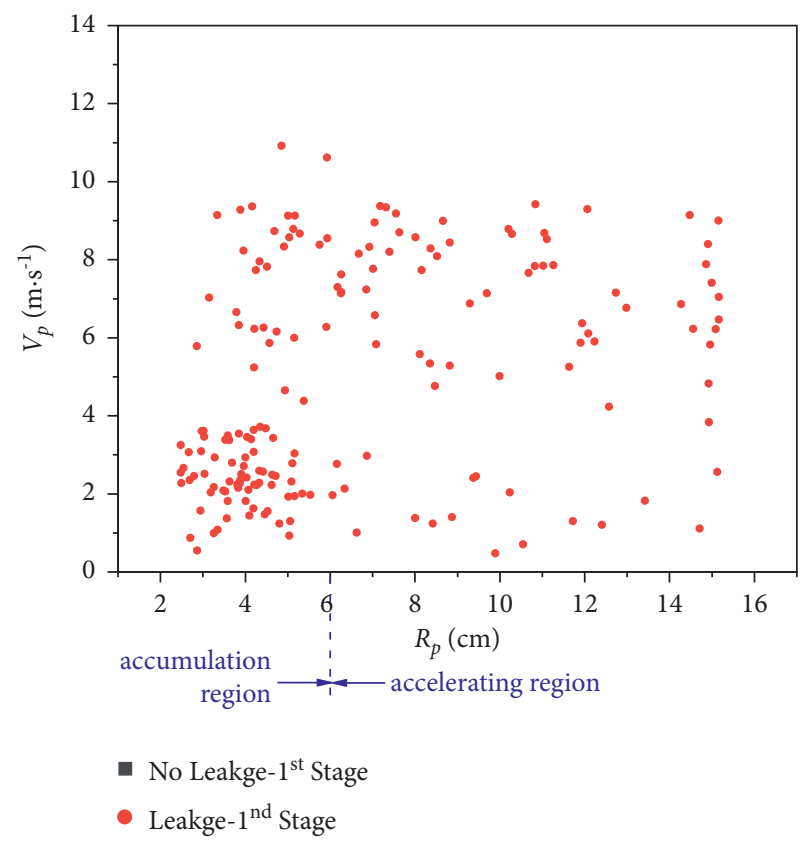

(a)

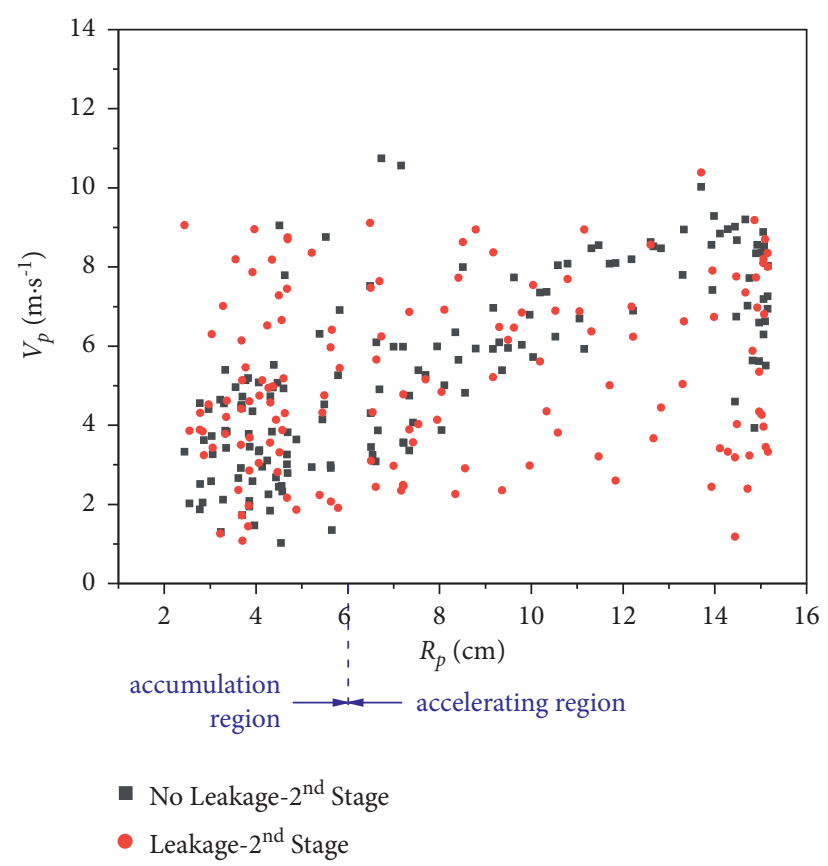

(b)

FIgURE 13: Particle velocity versus particle radial position in the impeller at $1.2 \mathrm{~s}$ at $Q=Q_{d}$ with or without leakage: (a) first stage and (b) second stage.

linearly with $R_{p}$. In the leak situation, particles are influenced by the vortex caused by the leakage flow, and $V_{p}$ is not related to $R_{p}$. At the near-wall region
$\left(R_{p}=14 \sim 16 \mathrm{~cm}\right), V_{p}$ decreases because of particle collision with the impeller wall. The linear relation between $V_{p}$ and $R_{p}$ weakened in the second stage in the nonleak case. 


\section{Conclusions}

In this study, the influence of the leakage flow on the motion characteristics of coarse particles in a double-stage slurry pump was studied by numerical simulation. The following conclusions are drawn:

(1) The proportion of leakage flow in the main flow $(Q L /$ Q) falls from $26 \%-27 \%$ to $8 \%-9 \%$ for the two stages. The flow rate increases from $80 \mathrm{~m}^{3} / \mathrm{h}$ to $200 \mathrm{~m}^{3} / \mathrm{h}$. Leakage flow from the front chamber $(Q L 2)$ and balance hole $(Q L 3)$ occupies almost the same portion in the first stage at a flow rate of less than $160 \mathrm{~m}^{3} / \mathrm{h}$.

(2) In the first-stage impeller, given the leakage flow squeeze from the balance hole, the streamline deviates to the shroud, and the vortex was located at the hub side of the impeller exit. The coarse particles with high inertia on the variation of their motion direction cost more time, leading to partial accumulation in the impeller, especially in the leakage case. Therefore, attention should be paid to slurry pumps' operation to avoid the block of the slurry pump.

(3) In the nonleak situation, the incremental average kinetic energy of particles in the two impellers is $130 \%$ and $104 \%$ of that in the leak situation, respectively. Compared with the particle count, the fluid suspension is the main factor in promoting the particles' kinetic energy in the impeller.

(4) More than $10 \%$ collision count in the acquired kinetic energy in the nonleak situation dissipates mainly in the bowl diffuser.

(5) No matter in the leak case or not, the average kinetic energy of particles was more than twice through the first stage but only 1.1 times through the second stage. The selection of stages in the slurry pump design should consider the limitation of particle velocity improvement.

\section{Data Availability}

The data used to support the findings of this study are included within the article.

\section{Conflicts of Interest}

The authors declare that they have no conflicts of interest.

\section{Acknowledgments}

This work was supported by the National Nature Science Foundation of China (52071296 and 51706206), Natural Science Foundation of Zhejiang Province (LZ15E090002 and LGF19E060001), Science Foundation of Zhejiang SciTech University (ZSTU) (19022101-Y), Top-notch Talent Support Program of Zhejiang Province (2019R51002), and Key Research and Development Program of Zhejiang Province (2020C01027).

\section{References}

[1] R. Tarodiya and B. K. Gandhi, "Hydraulic performance and erosive wear of centrifugal slurry pumps - a review," Powder Technology, vol. 305, pp. 27-38, 2017.

[2] K. C. Wilson, G. R. Addie, A. Sellgren, and R. Clift, Slurry Transport Using Centrifugal Pumps, Springer Science \& Business Media, Berlin, Heidelberg, Germany, 2006.

[3] B. Shi, J. j. Wei, and Y. Zhang, "Phase discrimination and a high accuracy algorithm for PIV image processing of particlefluid two-phase flow inside high-speed rotating centrifugal slurry pump," Flow Measurement and Instrumentation, vol. 45, pp. 93-104, 2015.

[4] R. Tarodiya and B. K. Gandhi, "Numerical simulation of a centrifugal slurry pump handling solid-liquid mixture: effect of solids on flow field and performance," Advanced Powder Technology, vol. 30, no. 10, pp. 2225-2239, 2019.

[5] R. Wang, Y. Guan, X. Jin, Z. Tang, Z. Zhu, and X. Su, "Impact of particle sizes on flow characteristics of slurry pump for deep-sea mining," Shock and Vibration, vol. 2021, Article ID 6684944, 13 pages, 2021.

[6] G. P. Glasby, J. Li, and Z. Sun, "Deep-sea nodules and Co-rich Mn crusts," Marine Georesources \& Geotechnology, vol. 33, no. 1, pp. 72-78, 2015.

[7] R. Sharma, "Environmental issues of deep-sea mining," Procedia Earth and Planetary Science, vol. 11, pp. 204-211, 2015.

[8] H. Zhu, J. Zhu, R. Rutter, and H.-Q. Zhang, "A numerical study on erosion model selection and effect of pump type and sand characters in electrical submersible pumps by sandy flow," Journal of Energy Resources Technology, vol. 141, no. 12, Article ID 122004, 2019.

[9] T. Engin and M. Gur, "Comparative evaluation of some existing correlations to predict head degradation of centrifugal slurry pumps," Journal of Fluids Engineering, vol. 125, no. 1, pp. 149-157, 2003.

[10] J. H. Liu and M. Y. Zhu, "Numeration simulation of solidliquid two-phase flow in centrifugal sewerage pump," Applied Mechanics and Materials, vol. 44-47, pp. 345-348, 2011.

[11] R. Tarodiya and B. K. Gandhi, "Numerical investigation of erosive wear of a centrifugal slurry pump due to solid-liquid flow," Journal of Tribology, vol. 143, no. 10, pp. 1-24, 2021.

[12] Y. Zhang, Y. Li, B. Cui, Z. Zhu, and H. Dou, "Numerical simulation and analysis of solid-liquid two-phase flow in centrifugal pump," Chinese Journal of Mechanical Engineering, vol. 26, no. 1, pp. 53-60, 2013.

[13] R. Tarodiya and B. K. Gandhi, "Effect of particle size distribution on performance and particle kinetics in a centrifugal slurry pump handling multi-size particulate slurry," Advanced Powder Technology, vol. 31, no. 12, pp. 4751-4767, 2020.

[14] C. Lei, Z. Yiyang, W. Zhengwei, X. Yexiang, and L. Ruixiang, "Effect of axial clearance on the efficiency of a shrouded centrifugal pump," Journal of Fluids Engineering, vol. 137, no. 7, 2015.

[15] T. Engin and M. Gur, "Performance characteristics of a centrifugal pump impeller with running tip clearance pumping solid-liquid mixtures," Journal of Fluids Engineering, vol. 123, no. 3, pp. 532-538, 2001.

[16] S. H. Park, The Effects of the Back Clearance Size and the Balance Holes on the Back Clearance Flow of the Centrifugal Pump with Semi-open Impeller, Texas A\&M University, TX, USA, 2008. 
[17] Y. Liu and L. Tan, "Spatial-temporal evolution of tip leakage vortex in a mixed-flow pump with tip clearance," Journal of Fluids Engineering, vol. 141, Article ID 081302, 2019.

[18] Y. Liu and L. Tan, "Theoretical prediction model of tip leakage vortex in a mixed flow pump with tip clearance," Journal of Fluids Engineering, vol. 142, Article ID 021203, 2020.

[19] Y. Liu, Y. Han, L. Tan, and Y. Wang, "Blade rotation angle on energy performance and tip leakage vortex in a mixed flow pump as turbine at pump mode," Energy, vol. 206, pp. 118104-118084, 2020.

[20] M. Patel, A. Kumar, B. Pardhi, and M. Pal, "Abrasive, erosive and corrosive wear in slurry pumps-A review," Int. Res. J. Eng. Technol, vol. 7, no. 3, pp. 2188-2195, 2020.

[21] Y. Li, H. Zhang, Z. Lin, Z. He, J. Xiang, and X. Su, "Relationship between wear formation and large-particle motion in a pipe bend," Royal Society open science, vol. 6, no. 1, Article ID 181254, 2019.

[22] X. Su, Z. Tang, Y. Li, Z. Zhu, K. Mianowicz, and P. Balaz, "Research of particle motion in a two-stage slurry transport pump for deep-ocean mining by the CFD-DEM method," Energies, vol. 13, no. 24, p. 6711, 2020.

[23] S. Huang, X. Su, and G. Qiu, "Transient numerical simulation for solid-liquid flow in a centrifugal pump by DEM-CFD coupling," Engineering Applications of Computational Fluid Mechanics, vol. 9, no. 1, pp. 411-418, 2015.

[24] M. Zhou, S. Wang, S. Kuang, K. Luo, J. Fan, and A. Yu, "CFDDEM modelling of hydraulic conveying of solid particles in a vertical pipe," Powder Technology, vol. 354, pp. 893-905, 2019.

[25] H. Xiao and J. Sun, "Algorithms in a robust hybrid CFD-DEM solver for particle-laden flows," Communications in Computational Physics, vol. 9, no. 2, pp. 297-323, 2011. 